\title{
The efficacy and safety of Oxaliplatin-Vinorelbine as a second-line chemotherapy combination in patients with platinum-resistant pretreated epithelial ovarian cancer: A retrospective study
}

\author{
Fidia Mumtahana, Xinli Tian, Teng Zhang, Baoxia Cui \\ Department of Obstetrics and Gynecology, Qilu Hospital of Shandong University, Jinan, Shandong Province, China
}

Received September 09, 2014; Revised October 08, 2014; Accepted October 09, 2014; Published Online October 10, 2014

\section{Original Article}

\begin{abstract}
Purpose: The main purpose of this study was to analyze the effects and tolerability of Oxaliplatin-Vinorelbine combination on Platinum-resistant epithelial ovarian carcinoma (EOC) patients. Methods: A single centered retrospective study comprising of 34 patients was conducted, and all 34 patients were treated with Vinorelbine $30 \mathrm{mg} / \mathrm{m}^{2}$ on day 1 and 8 along with Oxaliplatin 100 $\mathrm{mg} / \mathrm{m}^{2}$ on day 1 of 3 weeks treatment cycle following progressive platinum-resistant EOC. Results: The combination showed an overall response rate (ORR) of $18 \%$ (95\% CI, 4.4 - 31.6) where 2 (6\%) patients had complete response and 4 (12\%) patients had partial response. Stable disease was observed in 9 (26\%) patients and progressive disease in $19(56 \%)$ patients. Median diseases free survival, median relapse free survival and median time to progression was 17.05 months, 4.4 months, and 1.25 months, respectively. Hematological toxicities were mild; only 1 (2.9\%) patient had G3 anemia and major non-hematological toxicities include nausea-vomiting, diarrhea, constipation, hepatotoxicity, fatigueness and alopecia, which are mainly limited to G1-G2 and reversible. Conclusion: The effect of this combination is moderate as a second line treatment of platinum resistant EOC; however, in comparison with other regimens of Vinorelbine and Oxaliplatin, the activity is substandard but the toxicity profile is well tolerable. Further multicenter evaluation is needed for the better understanding of the therapeutic efficacy of the combination.
\end{abstract}

Keywords: Oxaliplatin-Vinorelbine Combination; Second-Line Chemotherapy; Platinum Resistant Epithelial Ovarian Cancer

\section{Introduction}

Ovarian cancer is the most fatal gynecologic carcinoma around the world.1, 2 Approximately 225,000 women are diagnosed for ovarian cancer per year and there is record of almost 1, 40,000 deaths worldwide out of this disease. ${ }^{3,4}$ Symptoms of the disease are not significant which may mimic other diseases conditions and cause delayed diagnosis. ${ }^{5}$ The majority of the women about $75 \%$ are diagnosed with advanced stage diseases that are FIGO III or IV and overall survival rate for 5 years is about $40 \% .^{6-10}$ The application of cytoreductive surgery followed by platinum-based chemotherapy, with or without the addition of a taxane is the most accepted treatment for advanced EOC. $3,11,12$ Record reveals that approximately $75 \%$ of these patients attain complete clinical remission after the initial treatment. ${ }^{4}$ Despite this fact, the majority of women with advanced EOC will ultimately relapse and $20 \%$ of these patients with platinum- and taxane-resistant disease. ${ }^{13-16}$ Cases of recurrences within 6 months following an initial response to platinum-based treatment or patients with stable disease during Platinum-based therapy are recognized as having platinum-resistant EOC. ${ }^{17}$ These very particular patients experience poor outcome and deserve to extend their survival expectancy with relieved symptoms with a better quality of life. ${ }^{13}$ So there is a necessity of second- line treatment to be administered for better physiological function and desired level of performance. At present diversive active second - line agents are available for ovarian cancer treatment and clinicians are using these frequently. ${ }^{15}$ But the result of these second-line treatments on survival is usually not upto the mark. ${ }^{18}$ Single as well as combination chemotherapy regimens are being used for the above mentioned patients. However, these regimens seem to cause greater level of toxicity without enhancing its efficacy. ${ }^{19,} 20$

Oxaliplatin (OXL) is a platinum derivative, diaminocyclohexane platinum which has exhibited activities contrary to 
different types of tumors with a symmetrical mechanism of impact like that of classic platinum drugs showing a different toxicity profile. Cisplatin or carboplatin seem not to be cross-resistant with OXL in human ovarian cell lines. A range of $16-29 \%$ is the response rate of oxaliplatin as a single acting agent. ${ }^{4}, 21,22$ Clinical result reveals no significant renal or auditive toxicity with oxaliplatin in recommended dose but marginally hematotoxicity being noticed. Acute cold-triggered dysesthesias with parasethesia and cumulative neurosensorial toxicity is the major frequent side effects of oxaliplatin. ${ }^{23-25}$

Vinorelbine (VNR) (5'-noranhydrovinblastine) is a semi synthetic anticancer agent derived from Vinca alkaloid group that exhibits an improved toxicity profile than vincristine and other Vinca alkaloid analogues and effective activity against advanced EOC. ${ }^{26,27}$ Application of VNR alone to patients with heavily pre-treated and platinum-resistant EOC has shown a $21 \%$ response rate. ${ }^{28,29}$ VNR projects a reduced level of neurotoxicity but causes a greater level of haematological toxicity. ${ }^{30,} 31$

Basing on the above mentioned information we decided to conduct a retrospective study on platinum pre-treated recurrent EOC patients to determine the efficacy and evaluate the toxicity of the combination of OXL-VNR regimen that is used in routine practice. To the best of our knowledge, there are not so much of studies available to determine the effects of this combination on platinum-resistant EOC patients.

\section{Patients and Methods}

\section{Patients}

Patient's data for this retrospective study was collected from the at the Department of Gynecology of Qilu Hospital of Shandong Medical University, China between July 2009 and March 2014.

Approval of the study was obtained from the local ethical committees and was conducted in accordance with the Declaration of Helsinki and Good Clinical Practice Guidelines.

The patient inclusion criteria: a) Histologically confirmed EOC; b) Relapsing disease within 6 months after platinum-containing combination therapy or progressive diseases while on treatment with measurable lesion (evidenced by imaging methods); c) Previously not treated with either OXL or VNR; d) Evidence of no other serious illness; e) Detailed clinicopathologic data and follow-up data. Medical records served with all the clinical information for every patient. Diseases status and required important data concerning the patients including tumor recurrence and patient death were provided from routinely maintained hospital registry.
TABLE 1: Patient characteristics.

\begin{tabular}{lcc}
\hline \hline Characteristics & No. of patients(n) & Percentage \% \\
\hline Patients evaluable & 34 & 100 \\
Age, years & 53 & \\
Median & $32-73$ & \\
Range & & \\
ECOG PS & 12 & 35 \\
0 & 16 & 47 \\
1 & 6 & 18 \\
2 & & \\
Tumor histology & 26 & 76 \\
Serous & 2 & 6 \\
Clear cell & 1 & 6 \\
Mucinous & 2 & 9 \\
Unknown & 3 & 100 \\
Poorly differentiated & & \\
Stage at diagnosis & 34 & \\
III C & & \\
\hline \hline
\end{tabular}

The patient exclusion criteria: a) Previous chemotherapy was not incorporated with platinum; b) Increased level of CA -125 was considered as the only indication of relapsing diseases; c) Having central nervous system metastasis; d) previous or existing carcinoma other than the ovarian.

A total 34 patients, aged between 32 and 73 years diagnosed with EOC and resistant to platinum based first line chemotherapy agents who were admitted in our department in above mentioned period and fulfilled the inclusion criteria are enrolled in this study. All the patients were diagnosed at FIGO stage IIIC. Table 1 comprises the patient characteristics. Prior to the administration of VNR-OXL regimen all the patients were administered with 234 Platinum derivative chemotherapy cycles (Table 2) with a median number of 2 cycles (range 1 to 8 ).

TABLE 2: Previous Platinum based chemotherapy regimen characteristics.

\begin{tabular}{lcc}
\hline \hline Characteristics & No. of patient & No. of cycles \\
\hline Chemotherapy cycles delivered & & \\
Median & & 2 \\
Range & & $1-8$ \\
Total & & 234 \\
No. of prior platinum-based & & \\
regimens to patients & & \\
1 & $25(73 \%)$ & \\
2 & $7(21 \%)$ & \\
3 & $2(6 \%)$ & \\
Chemotherapy regimens & & \\
Cisplatin+Cyclophosphamide & 10 & $70(30 \%)$ \\
Carboplatin+Paclitaxel & 13 & $65(27.9 \%)$ \\
Carboplatin+Docetaxel & 5 & $35(15 \%)$ \\
Cisplatin+Paclitaxel & 7 & $24(10.30 \%)$ \\
Cisplatin+Docetaxel & 3 & $15(6.43 \%)$ \\
Cisplatin+Topotecan & 4 & $14(6 \%)$ \\
Lobaplatin+Docetaxel & 1 & $6(2.57 \%)$ \\
Carboplatin+Topotecan & 1 & $4(1.7 \%)$ \\
Cisplatin+5FU & 1 & $1(0.43 \%)$ \\
\hline \hline
\end{tabular}


Prior to the treatment administration all eligible patients were confirmed with diseases relapse by evaluating clinical examination, serological tests and imaging modules.

\section{Given treatment}

VNR $30 \mathrm{mg} / \mathrm{m}^{2}$ was diluted in $100 \mathrm{ml}$ normal saline and administered as a 15 minutes intravenous infusion on days 1 and 8 of every 21-day treatment cycle. Again OXL $100 \mathrm{mg} / \mathrm{m}^{2}$ diluted in $500 \mathrm{ml}$ of $5 \%$ glucose solution and given as an intravenous infusion over 2 hours on day 1 which had started 15 minutes after the end of day 1 VNR administration. G-CSF was given prophylactically on days 12-16. All patients got standard antiemetic drugs and corticosteroids. Cycles were repeated every 3 weeks and treatment was continued unless diseases progression, toxicities were uncontrolled by conservative treatment. For an obvious delayed treatment, 25\% dosage for both drugs in subsequent cycles needed to be minimized.

Treatment characteristics of VNR-OXL regimen are shown in Table 3. A total 108 cycles of chemotherapy (with a median of 3) were administered. Median interval from previous treatment was 3.6 months (range: 0.7- 5.5 months). During the course of this regimen no treatment-related death was observed.

TABLE 3: Treatment characteristics.

\begin{tabular}{lc}
\hline \multicolumn{2}{c}{ Characteristics } \\
\hline Interval from previous treatment (months) \\
Median & 3.6 \\
Range & $0.6-5.4$ \\
Chemotherapy cycles delivered & 3 \\
Median & $1-7$ \\
Range & 108 \\
Total & \\
Interval between cycles (days) & 21 \\
Median & $21-42$ \\
Range & \\
Treatment duration (weeks) & 12 \\
Median & $3-31$ \\
Range & No. of patients \\
No. of cycles received by patients & $18(53 \%)$ \\
1-3 cycles & $11(32.3 \%)$ \\
4-5 cycles & $5(14.7 \%)$ \\
6-7 cycles & 82 \\
Percentage of cycles with full dose for both drugs & 26 \\
Percentage of cycles with delay (>24 days) & \\
Dose intensity mg/m ${ }^{2}$ (range) & $(22.5-30)$ \\
Vinorelbine & $(75-100)$ \\
Oxaliplatin & \\
\hline \hline
\end{tabular}

\section{Evaluation}

Prior to each course, patient's history and clinical examination with assessment of evaluable lesions, performance status, complete blood count along with other blood biochemistries (serum creatinine, serum bilirubin, AST, ALT, Alkaline phosphatase, serum electrolytes, calcium, magnesium, serum protein levels, Ca 125 assay) and ECG were performed.
Complete blood counts were done every weekly to assess the hematological toxicity, while imaging modules were carried out every three cycles for lesion assessment.

To determine the response rate and toxicity of the treatment World Health Organization (WHO) criteria for treatment response and toxicity were applied. ${ }^{32}$ The Kaplan-Meier method was selected for statistical analysis to determine median and confidence interval and for other data analysis.

\section{Results}

\section{Toxicity}

All the patients were evaluable for toxicity which is shown in Table 4. The main toxicity includes hematological toxicities, nausea-vomiting, diarrhea, constipation, hepatotoxicity, alopecia and fatigueness. $44 \%$ of the patients had anemia and among this G1 was commonly seen (29.4\%). 44\% and 35.3\% patients had Leukopenia and neutropenia respectively which remain within G1 and G2. 70.6\% patients suffered from nausea and vomiting but mainly G1(61.8\%). Diarrhea and constipation were observed in $41.15 \%$ and $32.2 \%$ patients respectively and in both G1 was common. $61.6 \%$ patient had alopecia but limited mainly within G1 and G2. Hepatotoxicity and fatigueness were seen in $32.2 \%$ (mainly G1) and 32.4 $\%$ (G2) patients respectively.

The minor toxicities were thrombocytopenia, febrile neutropenia, cardiac toxicity, nephrotoxicity, neurotoxicity, infections and skin rash which are mainly seen with G1 and G2. However, no toxicity reached to the level of G4. Due to hematological or extra-hematological toxicities 6 (17.6\%) patients had delayed treatment and $4(12 \%)$ had to go through dose reduction.

TABLE 4: Toxicity percentage in patients.

\begin{tabular}{lcccc}
\hline \hline Toxicity & Grade I & Grade II & Grade III & Grade IV \\
\hline Anemia & $10(29.4 \%)$ & $4(11.8 \%)$ & $1(2.9 \%)$ & \\
Leukopenia & $13(38.2 \%)$ & $2(5.8 \%)$ & - & - \\
Neutropenia & $7(20.6 \%)$ & $5(14.7 \%)$ & - & - \\
Thrombocytopenia & $8(23.5 \%)$ & - & - & - \\
Febrile neutropenia & $1(2.9 \%)$ & $3(8.8 \%)$ & - & - \\
Nausea / Vomiting & $18(53 \%)$ & $3(8.8 \%)$ & $3(8.8 \%)$ & - \\
Diarrhoea & $11(32.35 \%)$ & $3(8.8 \%)$ & - & - \\
Constipation & $8(23.5 \%)$ & $2(5.8 \%)$ & $1(2.9 \%)$ & - \\
Hepatotoxicity & $9(26.4 \%)$ & $1(2.9 \%)$ & $1(2.9 \%)$ & - \\
Cardiac toxicity & $2(5.8 \%)$ & $1(2.9 \%)$ & - & - \\
Nephrotoxicity & $5(14.7 \%)$ & - & - & - \\
Neurotoxicity & $4(11.8 \%)$ & $1(2.9 \%)$ & - & - \\
Alopecia & $12(35.2 \%)$ & $7(20.6 \%)$ & $2(5.8 \%)$ & - \\
Fatigue & - & $11(32.4 \%)$ & - & - \\
Infection & - & $4(11.8 \%)$ & - & - \\
Skin rash & - & $2(5.8 \%)$ & - & - \\
\hline \hline
\end{tabular}




\section{Response}

Among the 34 patients ORR was 18\% (95\% CI, 4.4 - 31.6); 2 (6\%) patients demonstrated CR to chemotherapy whereas 4 (12\%) patients showed PR (Table 5). Again 9 (26\%) of the patients had SD where the other 19 (56\%) patients had PD. The main sites of recurrence were abdomen and pelvic region and 7 patients had extra abdominal metastasis. All this progressive disease patients had 1-3 cycles and only one patient had 4 cycles of chemotherapy and subsequently switched over to other treatment after experiencing diseases progression. A clinical benefit (objective responses + stable disease) of $44 \%$ (95\% CI, 15.5 - 72.4) was observed in 15 patients within 34 patients.

TABLE 5: Response rate.

\begin{tabular}{lcc}
\hline \hline \multicolumn{1}{c}{ Response } & Patient no.(n=34) & Percentage \\
\hline Complete response(CR) & 2 & 6 \\
Partial Response (PR) & 4 & 12 \\
Overall response & 6 & 18 \\
$\quad$ rate (ORR) & & \\
Progressive disease & 19 & 56 \\
Stable disease & 9 & 26 \\
Clinical benefit & 15 & 44 \\
\hline \hline
\end{tabular}

\section{Survival and disease progression}

After a median follow-up of 3.1 months (range 0.2-21.3 months), median DFS was 17.05 months with a range of 12.8 to 21.3 months (95\% CI,-29.1 to 55.2). One patient with CR who was under our follow-up was dead uneventfully after a DFS of 21.3 months. Another patient with CR was under follow-up till her DFS of 12.8 months and then got lost. Again median RFS was 4.4 months with a range of 2.9 to 21.3 months (95\% CI, 0.65-15.8). 32 patients demonstrated disease progression.

TABLE 6: Time to progression and survival data.

\begin{tabular}{ll}
\hline \hline \multicolumn{2}{c}{ Months } \\
\hline Follow up & $0.2-21.3$ \\
Range & 3.1 \\
Median & \\
Disease free survival (DFS) \\
Events & 2 \\
Range & $12.8-21.3$ \\
Median & 17.05 \\
95\% CI & -29.1 to 55.2 \\
Relapse free survival (RFS) & \\
Events & 6 \\
Range & $2.9-21.3$ \\
Median & 4.4 \\
$95 \%$ CI & $0.65-15.8$ \\
Time to progression (TTP) & \\
Events & $32 / 34$ \\
Range & $0.8-2.9$ \\
Median & 1.25 \\
$95 \%$ CI & $0.98-1.4$ \\
\hline \hline
\end{tabular}

The median TTP was 1.2 months where the range was 0.8 2.9 months (Table 6) and (Figure 1). But obtaining the overall survival (OS) was not possible as except the 2 patients those who gained CR, other 32 patients went through different chemotherapy regimens on being determined with diseases progression.

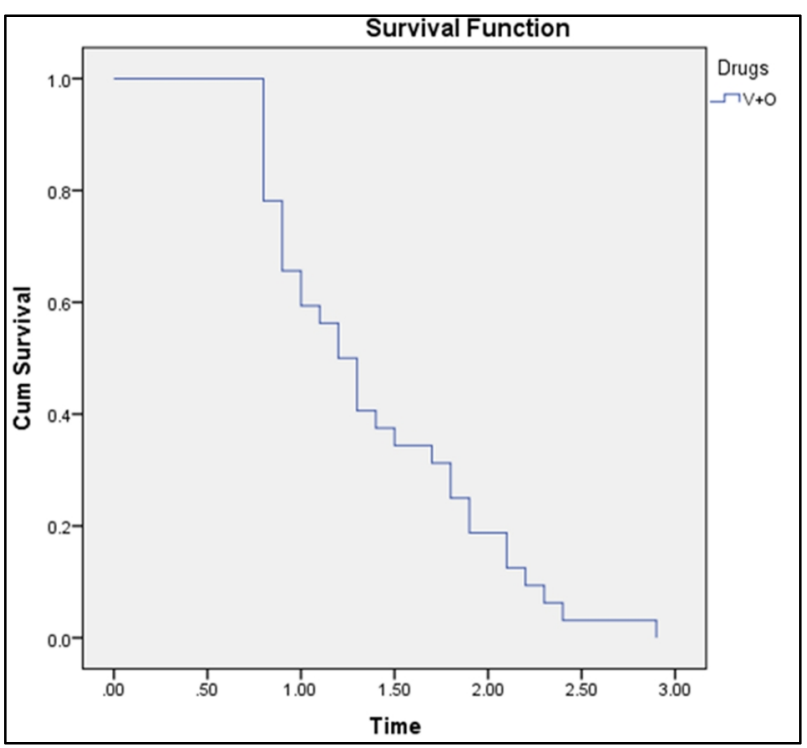

FIG. 1: Median TTP

\section{Discussion}

Despite the remarkable achievements in the last decades, most of the patients experienced diseases recurrence and platinum resistance after the standard treatment of EOC. ${ }^{33} \mathrm{~A}$ variety of chemotherapy agents are being applied for this purpose and among this OXL and VNR has shown promising activities against different types of carcinoma. ${ }^{34}$ Both of these drugs exhibited the opposite toxicity profile. ${ }^{25}$, 29 All these facts made us interested to determine the effects of the combination of these two drugs.

Total 34 patients aged between 32 and 73 years, were heavily pretreated with various types of platinum based chemotherapy with a number of 234 cycles following diagnosis at FIGO stage IIIC, developed platinum resistance and enrolled in our study with satisfactory inclusion criteria. After a median interval of 3.6 months from previous platinum based treatment they were further treated with OXL and VNR combination with a dose of $100 \mathrm{mg} / \mathrm{m}^{2}$ and $30 \mathrm{mg} / \mathrm{m}^{2}$ respectively. After getting 108 cycles with a median of 3 cycles, ORR was $18 \%$ (95\% CI, 4.4-31.6) that is 6 patients; $2(6 \%)$ with CR and $4(12 \%)$ with PR. SD was seen in $9(26 \%)$ patients and Progressive diseases were seen in $19(56 \%)$ patients. Out of 34 patients clinical benefit is seen in 15 (44\%) of them. Phase I study of VNR $\left(20-27 \mathrm{mg} / \mathrm{m}^{2}\right)$ and OXL $\left(40-55 \mathrm{mg} / \mathrm{m}^{2}\right)$ on advanced solid tumors showed an ORR of $24 \%$ where CR was $1(4 \%)$, PR was $5(20 \%)$ and SD was $4(16 \%)$ as a second line chemotherapy combination. ${ }^{35}$ Again $21 \%$ ORR was observed 
in single agent Phase II study of VNR $\left(30 \mathrm{mg} / \mathrm{m}^{2}\right)$ and $17 \%$ in OXL (median dose of $\left.100 \mathrm{mg} / \mathrm{m}^{2}\right){ }^{24,36}$ In different Phase II study of VNR in combination like- VNR $\left(25 \mathrm{mg} / \mathrm{m}^{2}\right)$ with Docetaxel showed an ORR of $23.9 \%$, SD of $34.8 \%$, PD of $30.4 \%$ and VNR $\left(30 \mathrm{mg} / \mathrm{m}^{2}\right)$ with PLD had an ORR of $37 \%$, SD of $10 \%$ and PD 53.5\%. ${ }^{27,37}$ Again, OXL (100 mg/m²) along with Docetaxel provided an ORR of $37.5 \%$ and SD $43.75 \%$, OXL $\left(50 \mathrm{mg} / \mathrm{m}^{2}\right)$ with PLD showed an ORR of $31.5 \%$ and SD of $42.1 \%{ }^{4}, 38 \mathrm{VNR}\left(30 \mathrm{mg} / \mathrm{m}^{2}\right)$ with Gemcitabine combination showed an ORR of $11 \%$ and SD of $24 \%$, PD $65 \% .{ }^{39}$ However, OXL $\left(100 \mathrm{mg} / \mathrm{m}^{2}\right)$ with Gemcitabine had the ORR of $37 \%$ and SD of $41 \%{ }^{40}$

The median DFS of this regimen was 17.05 months (95\% CI, -29.1 to 55.2) with an optimum of 21.3 months after a median follow up of 3.1 months. Phase II study of VNR with Docetaxel showed a median DFS of 13 months. ${ }^{37}$ Median RFS of the combination was 4.4 months ( $95 \%$ CI, 0.65 - 15.8) where in Phase II study of VNR with docetaxel had 5 months and OXL-Gemcitabine combination showed 6.8 months. ${ }^{37,40}$ median TTP of VNR-OXL was 1.25 months (95\% CI, 0.98 1.4). Median TTP of VNR as a single agent was 3.1 months in Phase II study but with Docetaxel and PLD it showed 4.5 months and 5.5 months respectively. ${ }^{27,36,37}$ OXL with PLD showed median TTP of 5.5 months. ${ }^{4}$

The toxicity profile of drug combination was well tolerable and reversible. Hematological toxicities were mild, only $1(2.9 \%)$ patient had G3 anemia, 2 (5.8\%) and 5(14.7\%) patients had G2 leukopenia and neutropenia respectively. G2 febrile neutropenia was observed in $3(8.8 \%)$ patients. Non-hematological toxicity includes mainly nausea-vomiting, diarrhea, constipation, hepatotoxicity, alopecia, fatigueness and most of them are limited to mild grades. $61.6 \%$ patients exhibited alopecia which was up to G2; only 2 (5.8\%) patients had G3 alopecia. G2 fatigueness was observed in $11(32.4 \%)$ patients. Nausea and vomiting were observed in $70.6 \%$ patients but mainly G1 (53\%). Mild neurotoxicity was observed in 5 patients and among them only 1(2.9\%) was experiencing G2. By analysis other studies of OXL and VNR it is seen that toxicity profile is better and similar with most of the studies $(4,27,37-40)$ and can be manageable with conservative treatments.

This combination is very uncommon. Since the patients became platinum resistant, this combination is used as an option. It is observed that when the application of few cycles could not make any desirable improvement, it led to diseases progression in all the patients (except for the case of complete remission) and ultimately patients were switched over to other kinds of treatment. Since all the patients were alive during this short period of treatment, the overall survival could not be determined. The result of the study is proved to be of an average standard as they are applied on the patients already treated with 1-3 platinum based chemotherapy regimens. But VNR and OXL when being combined with other chemotherapy agents, the effect seems to be better than this combination but the toxicity profile approximately remains similar with the safe margin.

\section{Conclusion}

The Oxaliplatin-Vinorelbine combination is a regimen with a moderate therapeutic effect but compared to other regimens of both drugs, the activity is substandard with a tolerable toxic side effect in patients with recurrent platinum registrant EOC. However, this retrospective analytical study suggests the need of further evaluation since it was limited to less number of patients which might be contributory designing the future study to evaluate its clinical utility. As such, more elaborate multicenter, prospective and randomized controlled clinical studies with a large number of patients are necessary to evaluate its therapeutic and side effects for the treatment of recurrent and platinum registrant EOC.

\section{Abbreviation}

Epithelial ovarian carcinoma (EOC), Vinorelbine (VNR), Oxaliplatin (OXL), Overall response rate (ORR), Complete response (CR), Partial response (PR), Stable diseases (SD), Progressive diseases (PD), Diseases free survival (DFS), Relapse free survival (RFS), Time to progression (TTP), Pegylated liposomal doxorubicin (PLD), Granulocyte colony-stimulating factor (G-CSF)

\section{Acknowledgement}

Authors would like to thank all the staffs of Department of Gynecology, Qilu Hospital of Shandong University for their support and all the patients involved in the study.

\section{Conflict of interest}

The authors declare that they have no conflicts of interest. The authors alone are responsible for the content and writing of the paper.

\section{References}

1. Miyake TM, Sood AK, Coleman RL. Contemporary use of bevacizumab in ovarian cancer. Expert Opin Biol Ther 2013; 13:283-94.

2. Poveda A, Ray-Coquard I, Romero I, et al. Emerging treatment strategies in recurrent platinum-sensitive ovarian cancer: focus on trabectedin. Cancer Treat Rev 2014; 40:366-75.

3. Della Pepa C, Banerjee S. Bevacizumab in combination with chemotherapy in platinum-sensitive ovarian cancer. OncoTargets and Therapy 2014; 7:1025-32. 
4. Salah-Eldin MA, Wahba HA, Halim AA. Biweekly peglated liposomal doxorubicin/oxaliplatin for ovarian cancer resistant to taxane-platinum treatment: a Phase II study. Indian J Cancer 2012; 49:169-75.

5. Aravantinos G, Pectasides D. Bevacizumab in combination with chemotherapy for the treatment of advanced ovarian cancer: a systematic review. J Ovarian Res 2014; 7:57.

6. Leamon CP, Lovejoy CD, Nguyen B. Patient selection and targeted treatment in the management of platinum-resistant ovarian cancer. Pharmgenomics Pers Med 2013; 6:113-25.

7. Hasan J, Jayson GC. Oral melphalan as a treatment for platinum-resistant ovarian cancer. Br J Cancer 2003; 88:1828-30.

8. Warschkow R, Tarantino I, Lange J, et al. Does hyperthermic intraoperative chemotherapy lead to improved outcomes in patients with ovarian cancer? A single center cohort study in 111 consecutive patients. Patient Safety in Surgery 2012; 6:12.

9. Kothari R, Argenta P, Fowler J, et al. Antiestrogen therapy in recurrent ovarian cancer resulting in 28 months of stable disease: a case report and review of the literature. Arch Oncol 2010; 18:32-5.

10. Jemal A, Siegel R, Ward E, et al. Cancer statistics, 2009. CA Cancer J Clin 2009; 59:225-49.

11. Kikkawa F, Nawa A, Ino K, et al. Advances in treatment of epithelial ovarian cancer. Nagoya $J$ Med Sci 2006; 68:19-26.

12. Kim A, Ueda Y, Naka T, Enomoto T. Therapeutic strategies in epithelial ovarian cancer. Journal of Experimental \& Clinical Cancer Research 2012; 31:14.

13. Sugiyama T. Second-line chemotherapy for platinum- and taxane-resistant epithelial ovarian cancer: Pegylated Liposomal Doxorubicin (PLD), irinotecan, and combination therapies at lower doses. Ovarian Cancer - Clinical and Therapeutic Perspectives, Dr. Samir Farghaly (Ed.) 2012; 14: 275.

14. Lee HJ, Kim HS, Park NH, et al. Feasibility of Oxaliplatin, Leucovorin, and 5-Fluorouracil (FOLFOX-4) Chemotherapy in Heavily Pretreated $\mathrm{Pa}$ tients with Recurrent Epithelial Ovarian Cancer. Cancer Res Treat 2013; 45:40-7.

15. Markman M, Bookman MA. Second-Line Treatment of Ovarian Cancer. The Oncologist 2000; 5:26-35.

16. Bellati F, Napoletano C, Gasparri ML, et al. Monoclonal antibodies in gynecological cancer: a critical point of view. Clin Dev Immunol 2011; 2011:890758.

17. Mantia-Smaldone GM, Edwards RP, Vlad AM. Targeted treatment of recurrent platinum-resistant ovarian cancer: current and emerging therapies. Cancer Manag Res 2011; 3:25-38.
18. Tomao F, Papa A, Rossi L, et al. Current status of bevacizumab in advanced ovarian cancer. Onco Targets Ther 2013; 6:889-99.

19. Coleman RL, Monk BJ, Sood AK, Herzog TJ. Latest research and treatment of advanced-stage epithelial ovarian cancer. Nat Rev Clin Oncol 2013; 10:211-24.

20. Sabbatini P, Spriggs D. Salvage therapy for ovarian cancer. Oncology (Williston Park) 1998; 12:833-43.

21. Monnet I, de CH, Soulié $\mathrm{P}$, et al. Oxaliplatin plus vinorelbine in advanced non-small-cell lung cancer: final results of a multicenter phase II study. Ann Oncol 2002; 13:103-7.

22. Faivre S, Kalla S, Cvitkovic E, et al. Oxaliplatin and paclitaxel combination in patients with platinum-pretreated ovarian carcinoma: An investigator-originated compassionate-use experience. Ann Oncol 1999; 10:1125-8.

23. Piccart MJ, Green JA, Lacave AJ, et al. Oxaliplatin or paclitaxel in patients with platinum-pretreated advanced ovarian cancer: A randomized phase II study of the European Organization for Research and Treatment of Cancer Gynecology Group. JClin Oncol 2000; 18:1193-202.

24. Chollet P, Bensmaïne MA, Brienza S, et al. Single agent activity of oxaliplatin in heavily pretreated advanced epithelial ovarian cancer. Ann Oncol 1996; 7:1065-70.

25. Misset JL. Oxaliplatin in practice. Br J Cancer 1998; 77 Suppl 4:4-7.

26. Cattel L, Passera R, Katsaros D, et al. Pegylated liposomal doxorubicin and vinorelbine in recurrent ovarian carcinoma: a pharmacokinetic study on alternate administration sequences. Anticancer Res 2006; 26:745-50.

27. Katsaros D, Oletti MV, Rigault de la Longrais IA, et al. Clinical and pharmacokinetic phase II study of pegylated liposomal doxorubicin and vinorelbine in heavily pretreated recurrent ovarian carcinoma. Ann Oncol 2005; 16:300-6.

28. Bajetta E, Di Leo A, Biganzoli L, et al. Phase II study of vinorelbine in patients with pretreated advanced ovarian cancer: activity in platinum-resistant disease. JClin Oncol 1996; 14:2546-51.

29. Tambaro R, Greggi S, Iaffaioli RV, et al. An escalating dose finding study of liposomal doxorubicin and vinorelbine for the treatment of refractory or resistant epithelial ovarian cancer. Ann Oncol2003; 14:1406-11.

30. Gregory RK, Smith IE. Vinorelbine--a clinical review. Br J Cancer 2000; 82:1907-13.

31. Goa KL, Faulds D. Vinorelbine. A review of its pharmacological properties and clinical use in cancer chemotherapy. Drugs Aging 1994; 5:200-34. 
32. Miller AB, Hoogstraten B, Staquet M, Winkler A. Reporting Results of Cancer Treatment. Cancer 1981; 47: 207-14.

33. Dogan NU, Schneider A, Chiantera V, et al. Tertiary cytoreduction in the setting of recurrent ovarian cancer (Review). Oncol Lett 2013; 6:642-47.

34. Harries M, Kaye SB. Recent advances in the treatment of epithelial ovarian cancer. Expert Opin Investig Drugs 2001; 10:1715-24.

35. Kakolyris S, Kouroussis C, Koukourakis M, et al. A dose-escalation study of oxaliplatin and vinorelbine in patients with advanced solid tumors. Oncology 2002; 63:213-8.

36. Sorensen P, Hoyer M, Jakobsen A, et al. Phase II study of vinorelbine in the treatment of platinum-resistant ovarian carcinoma. Gynecol Oncol 2001; 81:58-62.

37. Aravantinos G, Bafaloukos D, Fountzilas G, et al. Phase II study of docetaxel-vinorelbine in platinum-resistant, paclitaxel-pretreated ovarian cancer. Ann Oncol 2003; 14:1094-9.

38. Wang J, Han N, Wang HL, et al. Therapeutic effect of docetaxel combined with oxaliplatin for treatment of recurrent epithelial ovarian cancer. Nan Fang Yi Ke Da Xue Xue Bao 2009; 29:2319-20.

39. Xenidis $\mathrm{N}$, Neanidis $\mathrm{K}$, Amarantidis $\mathrm{K}$, et al. Biweekly vinorelbine and gemcitabine as second-line and beyond treatment in ovarian cancer. Cancer Chemother Pharmacol 2011; 67:69-73.

40. Vici P, Sergi D, Pizzuti L, et al. Gemcitabine-oxaliplatin (GEMOX) as salvage treatment in pretreated epithelial ovarian cancer patients. J Exp Clin Cancer Res 2013; 32:49. 